\title{
Neoadjuvant therapy in borderline resectable pancreatic ductal adenocarcinoma: A single institution review
}

\author{
Shilpen A. Patel ${ }^{1}$, Sanjay Chandrasekaran ${ }^{1}$, Gary N. Mann², Suchitra Chandrasekaran ${ }^{3}$, Felicia R. \\ Lucas $^{1}$, Edward Y. Kim¹, Andrew L. Coveler ${ }^{4}$ \\ 1. Department of Radiation Oncology, University of Washington School of Medicine, Seattle, WA, USA. 2. Department of \\ Obstetrics and Gynecology, Hospital of the University of Pennsylvania, Philadelphia, PA, USA. 3. Division of Medical \\ Oncology, University of Washington School of Medicine, Seattle, WA, USA. 4. Division of General Surgery, University of \\ Washington School of Medicine, Seattle, WA, USA.
}

Correspondence: Shilpen A. Patel, MD. Address: Department of Radiation Oncology, The University of Washington School of Medicine, 1959 NE Pacific St. Box 356043, Seattle, WA 98195, USA. Email: shilpenp@uw.edu

Received: August 22, 2014

DOI : $10.5430 /$ jst.v4n4p1

Online Published: December 9, 2014

Accepted: November 24, 2014

URL: http://dx.doi.org/10.5430/jst.v4n4p1

\section{Abstract}

Objectives: Margin negative (R0) resection is the only known curative therapy for Pancreatic Ductal Adenocarcinoma (PDA), but only $20 \%$ of cases are resectable. Neoadjuvant therapy (NATx) in borderline resectable PDA may increase chance for R0 resection and improve overall survival (OS).

Methods: We retrospectively identified 52 patients with borderline resectable PDA based on radiologic findings of clear celiac axis margins and no distant disease with local involvement of the superior mesenteric vein, portal vein, hepatic artery, gastroduodenal artery, or superior mesenteric artery $\leq 180$ degrees.

Results: Patients were grouped based on NATx and resection history: 17 (33\%) NATx and resection (NATxR), 22 (42\%) NATx without resection (NATxO), and 13 (25\%) upfront resection (UR). Comparison of pathologic vs. clinical stage demonstrated a $41 \%$ rate of pathologic downstaging in the NATxR group vs. $77 \%$ rate of clinical under staging in the UR group. Median lymph node ratio (number of positive nodes vs. total number of nodes harvested), was lower in the NATxR vs. UR group ( $0 \%$ vs. $12.5 \%$ ). R0 resection correlated with NATx (NATxR 71\% vs. UR 31\%; $p=.003$ ). Radiation therapy during NATx was associated with an even higher R0 resection rate ( $82 \%$ vs. $25 \% ; p=.05)$. Median OS in months was highest in the NATxR group (40.7) vs. UR (22.8), and lowest in unresected patients (13.4) ( $p=.0002)$.

Conclusions: NATx in borderline resectable PDA can abate disease progression and increase chance of R0 resection. NATxR and surgical resection leads to the best OS in this analysis.

\section{Key words}

Pancreatic ductal adenocarcinoma, Borderline resectable, Neoadjuvant therapy, Margin negative resection

\section{Introduction}

Pancreatic ductal adenocarcinoma (PDA) is the $4^{\text {th }}$ most common cause of cancer related deaths in the United States and median overall survival (mOS) is poor, even for resectable (American Joint Committee on Cancer [AJCC] Stage I or II) 
disease, ranging from 17 to 23 months ${ }^{[1,2]}$. Margin negative (R0) surgical resection is the only curative treatment, however only $20 \%$ of patients present with resectable disease ${ }^{[1]}$. In 2006, Varadhachary and colleagues from MD Anderson proposed a radiologic classification system for differentiating surgical resectability into resectable, borderline, and locally-advanced PDA using computer-tomography (CT) imaging ${ }^{[3]}$. More recent criteria have proposed consideration of other clinical correlates, including known loco-regional lymph node metastases, elevated CA 19-9 levels, marginal patient performance status, or severe pre-existing co-morbidities precluding surgery ${ }^{[4,5]}$. There is no unanimously accepted guideline for definition of borderline resectable PDA. According to the National Comprehensive Cancer Network $(\mathrm{NCCN})$ guidelines, borderline resectable PDA is primarily characterized by local involvement of the nearby vasculature, including the superior mesenteric vein (SMV), portal vein (PV), hepatic artery (HA), gastroduodenal artery (GDA), or superior mesenteric artery (SMA), with concurrent sparing of the celiac axis and no distant disease ${ }^{[4,6,7]}$.

Clinical management for patients with borderline resectable PDA is controversial. Recent data suggests neoadjuvant chemotherapy (NACT) or neoadjuvant chemoradiation (NACRT) prior to resection can facilitate disease downstaging, increase chance of R0 resection, reduce rates of locoregional occurrence, and ultimately lead to increases in overall survival ${ }^{[4,8-10]}$. The NCCN guidelines support neoadjuvant chemotherapy in borderline resectable PDA but do not provide therapeutic guidance.

However data from recent retrospective studies and single-arm prospective trials suggest a superior survival benefit with neoadjuvant gemcitabine-based regimens, especially in the setting of concurrent chemoradiation, versus alternate regimens with 5-Fluorouracil (5-FU) or paclitaxel ${ }^{[7,10-13]}$. The measurable benefit of neoadjuvant radiation therapy (NART) remains unclear. A multi-year analysis of data from the Surveillance, Epidemiology, and End Results (SEER) database in patients with Stage I or II disease indicated a 6 month increase in median overall survival in patients receiving NART vs. adjuvant RT ${ }^{[14]}$. In one of the largest single-institution reviews of 160 patients with borderline resectable disease, mOS across all patients was 18 months. Median OS in patients having neoadjuvant chemoradiation and resection was 40 months vs. 13 months in those that did not undergo resection ${ }^{[4]}$.

We aimed to retrospectively assess the benefit of NACT and NACRT in the setting of borderline resectable PDA at a large, academic medical center.

\section{Materials and methods}

\subsection{I nclusion criteria}

The University of Washington Medical Center (UWMC), Seattle Cancer Care Alliance (SCCA), and Fred Hutchinson Cancer Research Center (FHCRC) Institutional Review Boards approved this retrospective review. Initial patient datasets were obtained from UWMC and SCCA. UWMC and SCCA databases were screened from February 1995 to August 2010 based on billing codes for "pancreatic mass". Additional SCCA patients were obtained from a prospectively maintained institutional pancreatic cancer database. All patients were screened by diagnosis and only patients with AJCC $7^{\text {th }}$ edition Stage I or II PDA were included. Chart data abstracted included date of birth, gender, race, date of diagnosis, tumor size and location, date of surgery, number of lymph nodes sampled, lymph node status, pathologic stage and grade, resection margin status, CA 19-9 levels, neoadjuvant and adjuvant chemotherapies, radiation therapies. Date of death was determined using the Social Security Death Index (SSDI). Exclusion criteria included incomplete chart history for diagnosis date, date of therapy initiation, date of surgery, or survival status.

\subsection{Determination of clinical stage and surgical resectability}

Diagnostic CT imaging results were reviewed and clinical stage and surgical resectability status was determined. Clinical stage was determined based on the AJCC $7^{\text {th }}$ edition criteria. Patients diagnosed and staged according to the AJCC $6^{\text {th }}$ edition criteria were re-staged accordingly ${ }^{[15]}$. 
Borderline disease was defined based on the NCCN guidelines ${ }^{[7,16]}$. Required criteria for classification of borderline resectable disease based on CT imaging included no distant metastases and at least one of the following: 1) Venous involvement of the SMV and/or PV showing tumor abutment with or without narrowing or short-segment occlusion of the lumen with proximal and distal sparing allowing for safe resection, 2) GDA encasement up to the HA with either short segment encasement or direct abutment of the HA, without extension to the celiac axis, 3) tumor abutment of the SMA $\leq 180^{\circ}$ of the circumference of the vessel wall. Resectable disease was determined based on radiologic identification of the following: 1) No SMV or PV abutment, encasement, or tumor thrombus, and 2) clear fat planes around the celiac axis, HA, and SMA. Unresectable disease was determined based on tumor imaging demonstrating 1) $>180^{\circ}$ SMA encasement, 2) celiac axis abutment, 3) SMV/PV occlusion not considered safe for resection or reconstruction or 4) aortic artery invasion.

\subsection{Data management and statistical analysis}

Charts were individually reviewed and abstracted for pertinent information. Survival status was confirmed using chart review and the online SSDI. Data management performed using Microsoft Access (Microsoft Corporation; Redmond, WA). Statistical analysis was performed using Stata 12.1 software (StataCorp LP; College Station, TX). Descriptive and analytic statistics were performed. Chi square and Fisher's exact tests were used to analyze categorical variables. Student T-test and Wilcoxon rank sum tests were performed for continuous variables based on normality. Shapiro Wilk and tests for skewness and kurtosis were performed to assess normality. Alpha error was set to 0.05 and all statistical analyses were 2-tailed. Kaplan-Meier survival analyses were performed using MedCalc statistical software (MedCalc Software bvba; Ostend, Belgium) to calculated OS and identify differences in mOS between subgroups.

\section{Results}

\subsection{Patient characteristics}

There were 1235 patients identified from the UWMC and SCCA datasets, of which 698 were confirmed for diagnosis of PDA. Complete chart histories were identified for 127 patients with non-metastatic (AJCC Stage I-III) PDA, of which 52 patients were identified as having borderline resectable disease (see Figure 1).

Figure 1. 1235 patients were identified for undergoing consultation of pancreatic mass. 698 were diagnosed with PDA. 52 (7\%) of patients with PDA were identified with borderline resectable PDA.

Note. NACT, Neoadjuvant Chemotherapy; NACRT, Neoadjuvant Chemoradiation; ACT, Adjuvant Chemotherapy; ACRT, Adjuvant Chemoradiation.

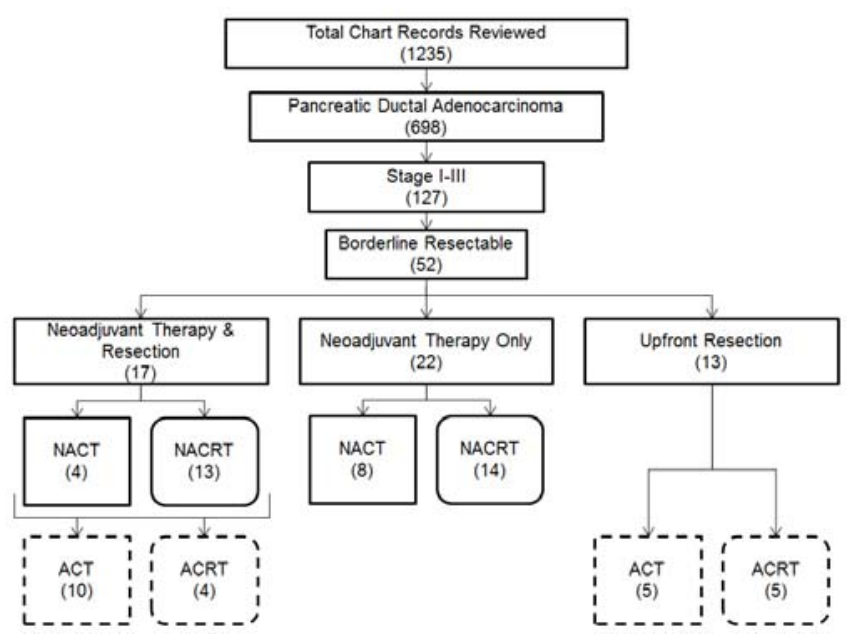

Patients with borderline resectable PDA were grouped into 3 categories. 17 patients received neoadjuvant therapy and successfully underwent surgical resection (NATxR), 22 patients received neoadjuvant therapy without completion of surgical resection (NATxO), and 13 patients underwent upfront resection without neoadjuvant therapy (UR). Median age at diagnosis for all 52 patients was 65 years (range 39-82) (see Table 1). Median age for patients in the NATxR group was lower than those patients undergoing upfront resection (60 vs. 71). Age was not normally distributed and no statistically 
significant difference in age was identified across the three groups according to Kruskal-Wallis test for non-parametric data $(p=.12)$. A total of 29 males and 23 females were included in the review. There were no significant differences in gender according to 2-tailed Fisher's exact test $(p=.60)$. Positive smoking history was noted on average from 59\%-73\% of patients across the 3 subject groups, with no statistically significant difference according to $\chi^{2}$ test $(p=.63)$.

Table 1. Demographic and Clinical Characteristics of 52 Borderline Resectable Patients Surveyed

\begin{tabular}{|c|c|c|c|c|}
\hline Characteristics & $\begin{array}{l}\text { Neoadjuvant Therapy } \\
\text { \& Resection } \\
\text { (17) }\end{array}$ & $\begin{array}{l}\text { Neoadjuvant Therapy } \\
\text { Only } \\
\text { (22) }\end{array}$ & $\begin{array}{l}\text { Upfront Resection } \\
(13)^{*}\end{array}$ & $p$-value \\
\hline \multicolumn{5}{|l|}{ Age -- yr } \\
\hline Median & 60 & 65 & 71 & .12 \\
\hline Range & $39-72$ & $43-82$ & $42-82$ & -- \\
\hline Male sex -- no. (\%) & $9(53)$ & $11(50)$ & $9(69)$ & .6 \\
\hline $\begin{array}{l}\text { Positive smoking Hx. -- } \\
\text { no. (\%) }\end{array}$ & $10(59)$ & $16(73)$ & $9(69)$ & .63 \\
\hline \multicolumn{5}{|l|}{ Clinical stage -- no. (\%) } \\
\hline IB & $1(6)$ & $1(5)$ & $6(46)$ & \\
\hline IIA & $9(53)$ & $11(50)$ & $6(46)$ & $.01^{\#}$ \\
\hline IIB & $7(41)$ & $10(45)$ & $1(8)$ & \\
\hline \multicolumn{5}{|l|}{ Tumor location -- no. (\%) } \\
\hline Head & $15(88)$ & $20(91)$ & $11(85)$ & \\
\hline Neck & $1(6)$ & $1(5)$ & $1(8)$ & -- \\
\hline Tail & $1(6)$ & $1(5)$ & 0 & \\
\hline \multicolumn{5}{|c|}{ Borderline anatomy characteristics -- no. (\%) } \\
\hline SMV Involvement & $9(53)$ & $13(59)$ & $4(31)$ & .28 \\
\hline PV Involvement & $10(59)$ & $9(41)$ & $10(77)$ & .13 \\
\hline
\end{tabular}

Abbreviations: SMV, Superior Mesenteric Vein; PV, Portal Vein.

*Tumor location unknown $(\mathrm{n}=1)$.

${ }^{\#}$ Statistically significant.

Eight patients (15\%) presented with Stage I disease vs. 44 (85\%) in Stage II. An increased prevalence of Stage I disease was noted in the UR group ( $p=.01$ ) (see Table 1). Of the 44 borderline patients with Stage II disease, $26(59 \%)$ were Stage IIA and 18 (41\%) Stage IIB. Tumor location was most commonly in the pancreatic head (Head: 46 (90\%); Neck: 3 (6\%); Tail: neck; $2(4 \%))$. Tumor location was unknown in one patient in the UR group. Radiologic findings involved the SMV and PV in $26(50 \%)$ and 29 (56\%) of the 52 borderline tumors, respectively. No statistically significant difference in SMV or PV involvement was noted across the three patient groups (SMV: $p=.28 ; \mathrm{PV}: p=.13$ ). Less commonly, tumors involved the GDA or HA (15\%), or SMA $<180$ degrees $(8 \%)$.

\subsection{Neoadjuvant chemotherapy}

Thirty-nine (75\%) of the 52 borderline patients received neoadjuvant therapy, of which 17 (43\%) successfully underwent resection (NATxR) and 22 (56\%) did not (NATxO). Median number of lines of chemotherapy given to patients in the NATxR was 2 lines (range 1-3) vs. 3 lines (range 1-6) in the NATxO group. Of the 17 resected patients, 5 (16\%) underwent surgical resection after one line of chemotherapy 11 (65\%) after two lines, and 1 (6\%) after 3 lines.

First line neoadjuvant chemotherapy in both groups most often included gemcitabine-based regimens (NATxR: 15 (89\%); NATxO: 19 (85\%)). The most commonly utilized first line was a combination of gemcitabine, taxotere, and capecitabine (GTX), given in 12 (71\%) and 13 (59\%) of NATxR and NATxO cases, respectively. In both groups, $2^{\text {nd }}$ line chemotherapy was most often capecitabine-based (NATxR: 10 [91\%]; NATxO: 11 [69\%]), with a combination of capecitabine and oxaliplatinmost commonly utilized in resected patients (NATxR: 8 [73\%]; NATxO: 5 [31\%]). 


\subsection{Neoadjuvant radiation therapy}

Thirteen (76\%) of NATxR patients vs. $14(64 \%)$ in the NATxO group received NART ( $p=.49)$. Median centigray (cGY) NART given was 3750 (range 3000-5050 cGY) in the NATxR group and 4460 (range 200-5580) in the NATxO group. In the NATxO group, specific NART dosing and scheduling details were unavailable for $2(14 \%)$ patients and 1 (7\%) patient received $200 \mathrm{cGY}$ emergent RT. NART was most often delivered during $2^{\text {nd }}$ line chemotherapy (NATxR: 10 (77\%); NATxO: $9(64 \%)$ ) vs. $1^{\text {st }}$ line (NATxR: 3 [23\%]; NATxO: 1 [7\%]). One (7\%) patient in the NATxO group received NART during $3^{\text {rd }}$ line chemotherapy. Of those 13 patients in the NATxR group that received NART, $8(62 \%)$ received NART concurrently with capecitabine and oxaliplatin.

\subsection{Surgical outcomes}

Resection was performed in a total of $30(58 \%)$ of the 52 borderline patients. Thirteen (25\%) underwent upfront resection, and of the 39 patients that received NATx, 17 (44\%) underwent resection. Mean number of lymph nodes sampled was 10.8 (95 CI 7.3 - 14.4) in the NATxR group and 13.46 (95 CI 9.4 - 17.5) in the UR group ( $p=.302$ ) (see Table 2). A lower rate of patients with positive lymph nodes was seen in the NATxR group vs. those receiving upfront resection (NATxR: 5 [20\%]; UR: 11 [85\%]; $p=.009$ ). Median lymph node ratio (LNR), as defined by the number of positive nodes vs. total number of lymph nodes harvested, was lower in the NATxR vs. UR group ( $0 \%$ [range $0-33$ ] vs. $12.5 \%$ [range 0-36]; $p=$ $.005)$. Nodes were not sampled in $2(12 \%)$ patients in the NATxR group at the time of resection.

Table 2. Surgical Outcomes in Resected Patients

\begin{tabular}{|c|c|c|c|}
\hline & $\begin{array}{l}\text { Neoadjuvant Therapy \& } \\
\text { Resection } \\
(17)^{*}\end{array}$ & $\begin{array}{l}\text { Upfront Resection } \\
\text { (13) }\end{array}$ & $p$-value \\
\hline No. of lymph nodes sampled -- mean (95 CI) & $10.8(7.3-14.4)$ & $13.5(9.4-17.5)$ & 0.302 \\
\hline \multicolumn{4}{|l|}{ Lymph node status -- no. (\%) } \\
\hline $\mathrm{N} 1$ & $5(29)$ & $11(85)$ & \multirow{2}{*}{$0.009^{\#}$} \\
\hline No & $10(59)$ & $2(15)$ & \\
\hline LNR -- median (range) & $0 \%(0-33)$ & $12.5 \%(0-36)$ & $0.005^{\#}$ \\
\hline \multicolumn{4}{|l|}{ pStage vs. cStage -- no. (\%) } \\
\hline$>$ & $4(24)$ & $10(77)$ & \multirow{3}{*}{--} \\
\hline$<$ & $7(41)$ & $3(23)$ & \\
\hline$=$ & $6(35)$ & 0 & \\
\hline R0 resection -- no. $(\%)$ & $12(71)$ & $4(31)$ & $0.003^{\#}$ \\
\hline
\end{tabular}

Abbreviations: LNR, Lymph Node Ratio; pStage, Pathologic Stage; cStage, Clinical Stage.

*Nodes not sampled $(\mathrm{n}=2)$.

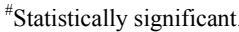

Pathologic stage (pStage) after resection was compared to clinical stage (cStage) at the time of diagnosis. Ten (77\%) of the 13 patients who underwent upfront resection were clinically understaged based on pathologic findings (pStage $>\mathrm{cStage}$ ), of which $9(90 \%)$ were due to pathologically positive lymph node disease (pN1 vs. cN0) and $1(10 \%)$ was due to metastatic disease. Seven (41\%) of patients in the NATxR group demonstrated downstaging, of which 4 cases (57\%) were due to node-downstaging (pN0 vs. $\mathrm{cN} 1$ ) and $3(43 \%)$ were due to reduction in tumor size. In resected patients receiving NATx, $12(71 \%)$ experienced R0 resection vs. $4(31 \%)$ in the UR group $(p=.003)$. Further analysis revealed that within the NATxR group, R0 resection was more often achieved in patients who received NACRT vs. NACT alone ( $82 \%$ vs. $25 \%$; $p$ $=.05)$.

\subsection{Adjuvant therapies}

Median number of lines of adjuvant chemotherapy given to all patients in the NATxR was 1 line (range 0-6) vs. 2 lines (range $0-5)$ in the UR group. In total, 24 (80\%) of resected patients received adjuvant chemotherapy (NATxR: 14 (82\%); 
UR: $10(77 \%))$. Of these patients, $8(57 \%)$ in the NATxR group received a single line of adjuvant chemotherapy vs. 3 $(30 \%)$ in the UR group.

First line adjuvant chemotherapy was most often gemcitabine-based (NATxR: 13 [93\%]; UR: 8 [80\%]). Adjuvant therapeutic regimen details were unavailable for one patient in both groups. Four (29\%) and $5(50 \%)$ of the patients received adjuvant radiation therapy (ART) in the NATxR and UR groups, respectively. No trends were identified regarding the utilization of ART between the two groups $(p=.44)$.

\subsection{Patient survival}

Overall survival across all patients ranged from 5.9 - 124.9 months (see Table 3). Patients in the NATxO group achieved median overall survivals of 13.4 months, whereas patients undergoing resection in the UR and NATxR groups achieved median survivals of 22.8 and 40.7 months, respectively $(p=.0002)$ (see Figure 2$)$. Eight $(47 \%)$ patients in the NATxR group were alive at the time of data analysis.

Table 3. Overall Survival

\begin{tabular}{llll}
\hline & Median OS (months) & Range mOS (months) & Alive at End Date no. (\%) \\
\hline Neoadjuvant therapy \& resection (17) & 40.7 & $12.9-124.9$ & $8(47)$ \\
Neoadjuvant therapy only (22)* & 13.4 & $5.9-63.6$ & $2(9)$ \\
Upfront resection (13) & 22.8 & $7.8-95.3$ & $2(15)$ \\
\hline
\end{tabular}

Abbreviations: mOS, Median Overall Survival.

*Exploratory laparotomy performed $(\mathrm{n}=8)$.

Figure 2. Kaplan Meier Survival Curves for patients receiving Neoadjuvant Therapy \& Resection, Neoadjuvant Therapy Only, and Upfront Resection.

*Statistically significant.

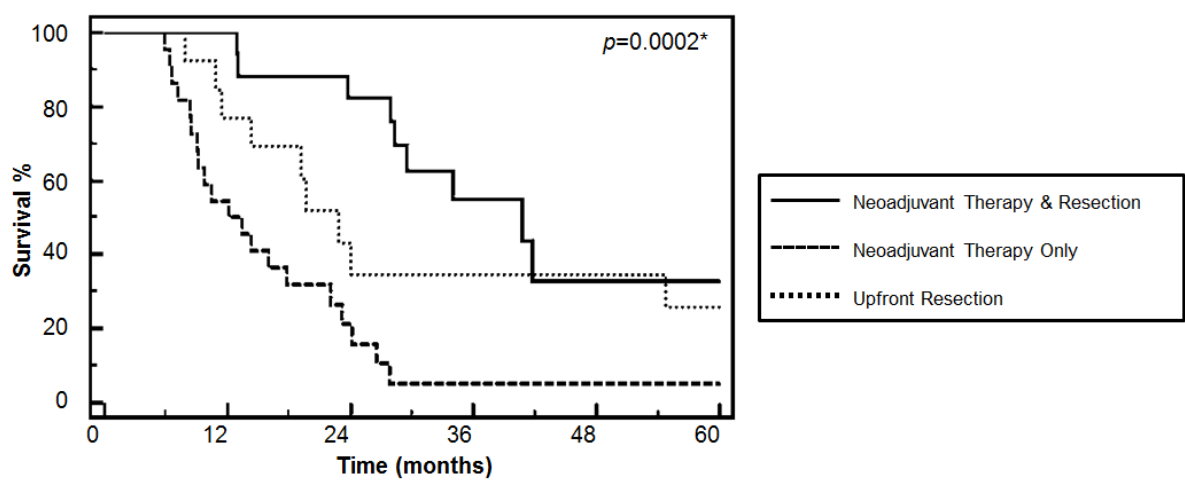

\section{Discussion}

We present a multi-year, retrospective, single-institution review of 52 patients treated for borderline PDA. Patients were stratified into 3 groups depending on history of neoadjuvant therapy and surgical resection. No significant differences across the three groups were identified regarding age, gender, smoking history, tumor location, or tumor anatomy (see Table 1). An increased prevalence of AJCC cStage I disease was identified in patients within the UR group (46\% [UR] vs. $6 \%$ [NATxR] vs. $5 \%$ [NATxO]).

Post-operative findings revealed neoadjuvant therapy prior to resection leads to decreased LNR, pathologic downstaging, and increased rates of $\mathrm{R} 0$ resection. The incidence of positive lymph nodes was reduced in those patients treated prior to resection ( $29 \%$ vs. $85 \%)$. Supporting this finding is that there was no significant difference in number of nodes sampled across both the NATxR and UR groups. Additionally, multiple retrospective reviews have indicated that the LNR is an 
independent prognostic factor vs. node status alone ${ }^{[17-19]}$. In our analysis, patients treated with neoadjuvant therapy prior to resection had a median LNR of $0 \%$ vs. $12.5 \%$ in the UR group.

Comparisons of AJCC cStage and pStage in resected patients demonstrated downstaging in 7 (41\%) of patients in the NATxR group. Of the 7 patients downstaged, 4 (57\%) were due to lymph node downstaging from cN1 to pN0 vs. 3 (43\%) due to downstaging in T-stage. This compares favorably vs. findings of clinical under staging (pStage $>$ cStage) in 10 (77\%) of patients in the UR group, of which $9(90 \%)$ were due to pathologic findings of node positive disease following node negative clinical staging. Despite our small sample size, these analyses and findings regarding the number of positive lymph nodes, LNR, and post-therapy AJCC pStage in borderline PDA are corroborated by other reports ${ }^{[17,19,20]}$. Our data encourages further study of the post-therapy pathologic benefits of neoadjuvant therapy, especially when recent data suggest the limited ability of radiologic criteria to identify post-therapy changes and neoadjuvant therapeutic success in borderline PDA ${ }^{[21]}$.

In our review, 17 (44\%) of the 39 patients who received neoadjuvant therapy underwent resection, 8 (21\%) underwent exploratory laparotomy and considered surgically unresectable, and $14(36 \%)$ did not demonstrate significant clinical response to therapy. Despite small sample size, our analyses compare favorably to the $41 \%$ overall resection rate and $16 \%$ unresectable rate reported by Evans et al in a 160 person study following neoadjuvant therapy and re-staging in patients with borderline PDA ${ }^{[4]}$. R0 resection is the only curative treatment for PDA, and we identified an increased rate of R0 resection in the NATxR group vs. UR group (71\% vs. 31\%). Within the NATxR group, those receiving NACRT experienced an increased rate of margin negative resection. Prior studies have also correlated completion of neoadjuvant therapy to increased rates of R0 resection, and our findings encourage additional investigation into quantifying the added benefits of neoadjuvant radiation therapy ${ }^{[22,23]}$.

Median overall survival in patients undergoing neoadjuvant therapy and resection in our review was 40.7 months in comparison to 13.4 months mOS in the NATxO group. These survival data are corroborated by the 40 months and 13 months mOS reported in similarly treated patient groups by Katz et al ${ }^{[4]}$. Patients in the UR group experienced mOS of 22.8 months. This nearly 2-fold difference in mOS between the NATxR and UR groups suggests a survival benefit associated with pre-operative neoadjuvant therapy and increased rate of $\mathrm{R} 0$ resection.,

This data is presented with certain limitations. Our sample population spans from 1995-2010, which includes patients prior to the $7^{\text {th }}$ edition AJCC staging guidelines thus requiring re-staging of some of the patients based on TNM findings and radiographic reports. Heterogeneity in patient treatment histories were accounted for based on analysis of chemotherapeutic regimens, with findings indicating that the majority of patients received $1^{\text {st }}$ line gemcitabine, which is generally accepted as the superior chemotherapy ${ }^{[10,11]}$. Our small sample size precluded multivariate-analysis and Cox regression, however the pathologic findings and survival outcomes we present parallel those of larger studies, thus suggesting clinically valuable findings.

In summary, PDA has poor patient prognosis and remains difficult to treat. R0 resection is the only curative option, and neoadjuvant therapy is a potential means for improving surgical outcomes. We believe aggressive neoadjuvant therapy in patients with borderline resectable PDA may result in increased chance of pathologic downstaging, R0 resection, favorable LNR, and improved overall survival. The addition of neoadjuvant radiation therapy may also contribute to increased chance of R0 resection. Additional prospective studies to validate these findings and improve patient outcomes in PDA are warranted.

\section{Acknowledgments}

The authors acknowledge the invaluable assistance of Joy Knopp, clinical research coordinator. The authors would like to recognize Phil K. Spady (UWMC) and Joyce Lau (SCCA) for providing patient datasets. 


\section{References}

[1] Vincent A, Herman J, Schulick R, Hruban RH, Goggins M. Pancreatic cancer. Lancet. 2004; 363(9414): $1049-1057$. http://dx.doi.org/10.1016/S0140-6736(04)15841-8

[2] Siegel R, Naishadam D, Jemal A. Cancer statistics, 2013. CA Cancer J Clin. 2013; 63(1): 11-30. PMid:23335087 http://dx.doi.org/10.3322/caac.21166

[3] Varadhachary GR, Tamm EP, Abbruzzese JL, et al. Borderline resectable pancreatic cancer: definitions, management, and role of preoperative therapy. Annals of Surgical Oncology. 2006; 13(8): 1035-1046. PMid:16865597 http://dx.doi.org/10.1245/ASO.2006.08.011

[4] Katz MHG, Pisters PWT, Evans DB, et al. Borderline resectable pancreatic cancer: the importance of this emerging stage of disease. J Am Coll Surg. 2008; 206(5): 833-46; discussion 846-8. PMid:18471707 http://dx.doi.org/10.1016/j.jamcollsurg.2007.12.020

[5] Evans DB, Erickson B a, Ritch P. Borderline resectable pancreatic cancer: definitions and the importance of multimodality therapy. Ann Surg Oncol. 2010; 17(11): 2803-5. PMid:20737218 http://dx.doi.org/10.1245/s10434-010-1285-8

[6] Springett GM, Hoffe SE. Borderline resectable pancreatic cancer: on the edge of survival. Cancer Control. 2008; 15(4): $295-307$.

[7] Tempero M, Arnoletti JP, Ben-Josef E, et al. Pancreatic adenocarcinoma: Clinical Practice Guidelines in Oncology. JNCCN. 2010; 8(9): 972-1017. PMid:20876541

[8] McClaine RJ, Lowy AM, Sussman JJ, Schmulewitz N, Grisell DL, Ahmad SA. Neoadjuvant therapy may lead to successful surgical resection and improved survival in patients with borderline resectable pancreatic cancer. HPB. 2010; 12(1): 73-9. PMid:20495649 http://dx.doi.org/10.1111/j.1477-2574.2009.00136.x

[9] Satoi S, Yanagimoto H, Toyokawa H, et al. Surgical results after preoperative chemoradiation therapy for patients with pancreatic cancer. Pancreas. 2009; 38(3): 282-288. PMid:19142173 http://dx.doi.org/10.1097/MPA.0b013e31819438c3

[10] Abbott DE, Baker MS, Talamonti MS. Neoadjuvant therapy for pancreatic cancer: a current review. J Surg Oncol. 2010; 101(4): 315-20. PMid:20187063 http://dx.doi.org/10.1002/jso.21469

[11] Varadhachary GR, Wolff RA, Crane $\mathrm{CH}$, et al. Preoperative gemcitabine and cisplatin followed by gemcitabine-based chemoradiation for resectable adenocarcinoma of the pancreatic head. J Clin Oncol. 2008; 26(21): 3487-95. PMid:18640929 http://dx.doi.org/10.1200/JCO.2007.15.8642

[12] Takai S, Satoi S, Yanagimoto H, et al. Neoadjuvant chemoradiation in patients with potentially resectable pancreatic cancer. Pancreas. 2008; 36(1): e26-e32. PMid:18192876 http://dx.doi.org/10.1097/mpa.0b013e31814b229a

[13] Poonacha TK, Go RS. Level of scientific evidence underlying recommendations arising from the National Comprehensive Cancer Network clinical practice guidelines. Journal of clinical oncology: official journal of the American Society of Clinical Oncology. 2011; 29(2): 186-91.

[14] Stessin AM, Meyer JE, Sherr DL. Neoadjuvant radiation is associated with improved survival in patients with resectable pancreatic cancer: an analysis of data from the surveillance, epidemiology, and end results (SEER) registry. Int J Radiat Oncol Biol Phys. 2008; 72(4): 1128-33. PMid:18538501 http://dx.doi.org/10.1016/j.ijrobp.2008.02.065

[15] Katz MHG, Hwang R, Fleming JB, Evans DB. Tumor-node-metastasis staging of pancreatic adenocarcinoma. CA Cancer J Clin. 2008; 58(2): 111-25. PMid:18272835 http://dx.doi.org/10.3322/CA.2007.0012

[16] National Comprehensive Cancer Network (NCCN). NCCN Clinical Practice Guidelines in Oncology. Pancreatic Adenocarcinoma. Available from: http://www.ncen.org. Accessed May 9, 2013.

[17] Berger AC, Watson JC, Ross EA, Hoffman JP. The metastatic/examined lymph node ratio is an important prognostic factor after pancreaticoduodenectomy for pancreatic adenocarcinoma. Am Surg. 2004; 70(3): 235-240; discussion 240. PMid:15055847

[18] Sierzega M, Popiela T, Kulig J, Nowak K. The ratio of metastatic/resected lymph nodes is an independent prognostic factor in patients with node-positive pancreatic head cancer. Pancreas. 2006; 33(3): 240-245. PMid:17003644 http://dx.doi.org/10.1097/01.mpa.0000235306.96486.2a

[19] Riediger H, Keck T, Wellner U, et al. The lymph node ratio is the strongest prognostic factor after resection of pancreatic cancer. $\mathrm{J}$ Gastrointest Surg. 2009; 13(7): 1337-44. PMid:19418101 http://dx.doi.org/10.1007/s11605-009-0919-2

[20] Estrella JS, Rashid A, Fleming JB, et al. Post-therapy pathologic stage and survival in patients with pancreatic ductal adenocarcinoma treated with neoadjuvant chemoradiation. Cancer. 2012; 118(1): 268-77. PMid:21735446 http://dx.doi.org/10.1002/cncr.26243

[21] Katz MHG, Fleming JB, Bhosale P, et al. Response of borderline resectable pancreatic cancer to neoadjuvant therapy is not reflected by radiographic indicators. Cancer. 2012; 118(23): 5749-56. PMid:22605518 http://dx.doi.org/10.1002/cncr.27636

[22] Gillen S, Schuster T, Meyer Zum Büschenfelde C, Friess H, Kleeff J. Preoperative/neoadjuvant therapy in pancreatic cancer: a systematic review and meta-analysis of response and resection percentages. PLoS Med. 2010; 7(4): e1000267. PMid:20422030 http://dx.doi.org/10.1371/journal.pmed.1000267

[23] Kang CM, Chung YE, Park JY, et al. Potential contribution of preoperative neoadjuvant concurrent chemoradiation therapy on margin-negative resection in borderline resectable pancreatic cancer. J Gastrointest Surg. 2012; 16(3): 509-17. PMid:22183861 http://dx.doi.org/10.1007/s11605-011-1784-3 